\title{
Manifestations dento-maxillo-faciales chez un enfant atteint d'une B-thalassémie majeure traitée par greffe de cellules souches hématopoïétiques. A propos d'un cas
}

\section{Oral features in a child with B-thalassemia major treated by haematopoietic stem cell transplantation. Report of a case}

SOPHIE JUNG (1), ANNELYSE GARRET-BERNARDIN (1), BRUNO GROLLEMUND (3), NADINE COJEAN (4), PATRICK LUTZ (4), MARIE-CÉCILE MANIÈRE (1), AHMED FÉKI (2)

\section{RÉSUMÉ}

Cet article présente le cas d'un garçon de 8 ans atteint de ß-thalassémie majeure, affection qui représente une forme d'anémie héréditaire grave. Le traitement initial par transfusions répétées a entraîné le développement d'une hémochromatose sévère mettant en jeu le pronostic vital. Le patient a bénéficié d'une greffe allogénique de cellules souches hématopoïétiques, seul traitement curatif de la thalassémie majeure, mais il a rapidement développé une maladie du greffon contre l'hôte.

Une prise en charge globale du patient a été réalisée dans le Département d'Odontologie Pédiatrique en raison des complications maxillo-faciales et bucco-dentaires importantes liées à ces deux affections. (Med Buccale Chir Buccale 2006; 12: 163-70).

mots clés : bêta-thalassémie, greffe de cellules souches hématopoïétiques, maladie du greffon contre l'hôte

\section{SUMMARY}

This paper is about an 8 year old boy suffering from B-thalassemia major which is a severe form of hereditary anaemia. The initial treatment by repeated transfusions had led to the development of haemochromatosis which engaged the vital prognosis. Thus, the patient received allogenic haematopoietic stem cell transplantation; the only curative treatment for thalassemia major, but he quickly developed a graft versus host disease.

A global management of the patient was performed in the Paediatric Dentistry Department because of the important maxillo-facial and oral repercussions of these pathologies. (Med Buccale Chir Buccale 2006; 12: 163-70).

key words: beta-thalassemia, haematopoietic stem cell transplantation, graft versus-host disease

1 - Département d'Odontologie Pédiatrique, Service de Soins Bucco-dentaires, Hôpitaux Universitaires de Strasbourg

2 - Département de Médecine Buccale et de Chirurgie Buccale, Service de Soins Bucco-dentaires, Hôpitaux Universitaires de Strasbourg

3 - Département d'Orthopédie Dento-Faciale, Service de Soins Bucco-dentaires, Hôpitaux Universitaires de Strasbourg

4 - Service de Pédiatrie III, Unité d’Hématologie-Oncologie, Hôpitaux Universitaires de Strasbourg

Demande de tirés à part :

Sophie Jung Faculté de Chirurgie Dentaire Département d'Odontologie Pédiatrique 1 place de l'Hôpital 67000 Strasbourg

e-mail : sojung@caramail.com 
médecine

buccale

chirurgie

buccale

VOL. $12, \mathrm{~N}^{\circ} 3$ 2006

page 164
La ß-thalassémie est une anomalie héréditaire de I'hémoglobine définie par une insuffisance de production de la chaîne $B$ de la globine [1]. Cette affection, surtout rencontrée dans le bassin méditerranéen, est responsable d'une dysérythropoïèse et d'une anémie hémolytique. La ß-thalassémie majeure ou anémie de Cooley, qui est la forme homozygote, présente les manifestations cliniques les plus sévères : retard de croissance, anomalies squelettiques, hépatosplénomégalie, susceptibilité accrue aux infections... Des transfusions sanguines régulières constituent le traitement de première intention. Elles permettent de limiter la survenue de complications mais elles entraînent souvent le développement d'une surcharge ferrique polyviscérale, c'est-à-dire d'une hémochromatose secondaire. Principal facteur de mortalité et de morbidité associé à la B-thalassémie [1], l'hémochromatose est à l'origine d'un trouble de croissance, d'un diabète bronzé, d'une cirrhose et surtout d'une cardiomyopathie. La greffe allogénique de cellules souches hématopoïétiques constitue le seul traitement réellement curatif de la ß-thalassémie car il permet la correction du défaut génétique touchant le système hématopoïétique [2].

D'importantes répercussions de la ß-thalassémie et de son traitement sont rencontrées dans le domaine bucco-dentaire et maxillo-facial et elles justifient une prise en charge au sein d'une équipe multidisciplinaire.

\section{OBSERVATION}

Un garçon d'origine turque, âgé de 8 ans, est pris en charge depuis sa naissance dans le Service d'Onco-hématologie Pédiatrique de Strasbourg pour une B-thalassémie majeure. II a été initialement traité par des transfusions mensuelles mais, à l'âge de 7 ans, il a développé une hémochromatose sévère engageant rapidement le pronostic vital (charge hépatique en fer s'élevant à 240 mmol.g-1). Le patient a ainsi bénéficié d'une allogreffe de cellules souches hématopoïétiques en février 1997 après un protocole de conditionnement composé de busulfan et d'endoxan. Le greffon, constitué de cellules souches périphériques, provenait d'un don- neur HLA identique mais non apparenté [3]. Cet enfant a rapidement développé une réaction du greffon contre l'hôte (GVHD pour Graft-versus- Host Disease) aiguë cutanéo-muqueuse de grade IV ${ }^{[4]}$ (Fig. 1 et 2), hépatique, digestive et pulmonaire de grade III dont l'évolution a été favorable sous corticoïdes, ciclosporine, tacrolimus et photothérapie externe avec des UVB [5]. En août 2005, l'enfant est ré-hospitalisé pour une GVHD cutanéo-muqueuse chronique avec des lésions lichénoïdes ; le diagnostic est confirmé par une biopsie cutanée. Cette forme extensive est traitée par corticoïdes, tacrolimus, sirolimus et par des séances de photophérèse extracorporelle [-1]].

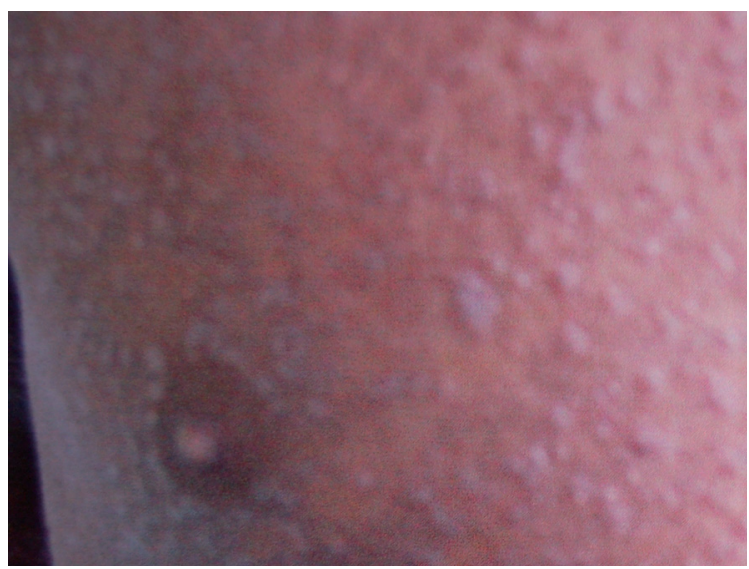

Figure 1 : Maladie du greffon contre l'hôte aiguë cutanée dans la région thoracique.

Acute thoracic cutaneous graft versus host disease.

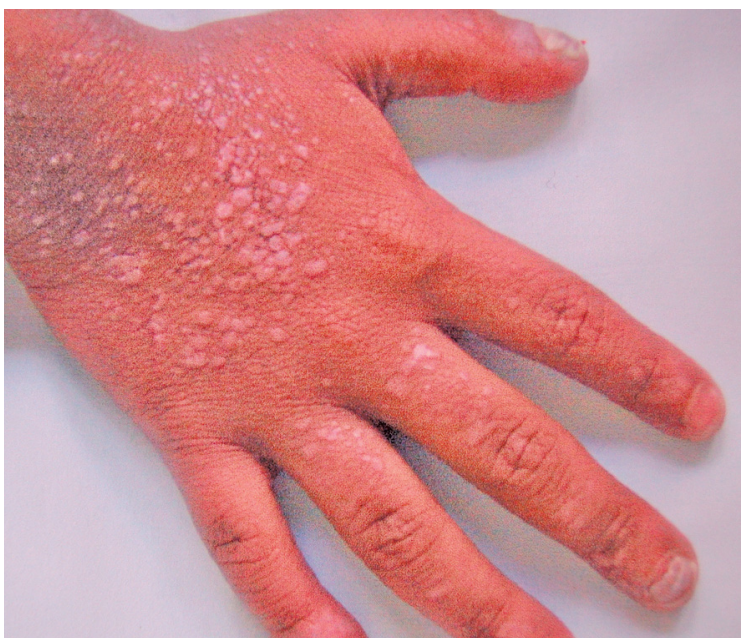

Figure 2 : Maladie du greffon contre l'hôte aiguë cutanée avec dyskératose unguéale ${ }^{[4]}$.

Acute cutaneous Graft versus Host Disease with ungueal dyskeratosis ${ }^{[4]}$. 


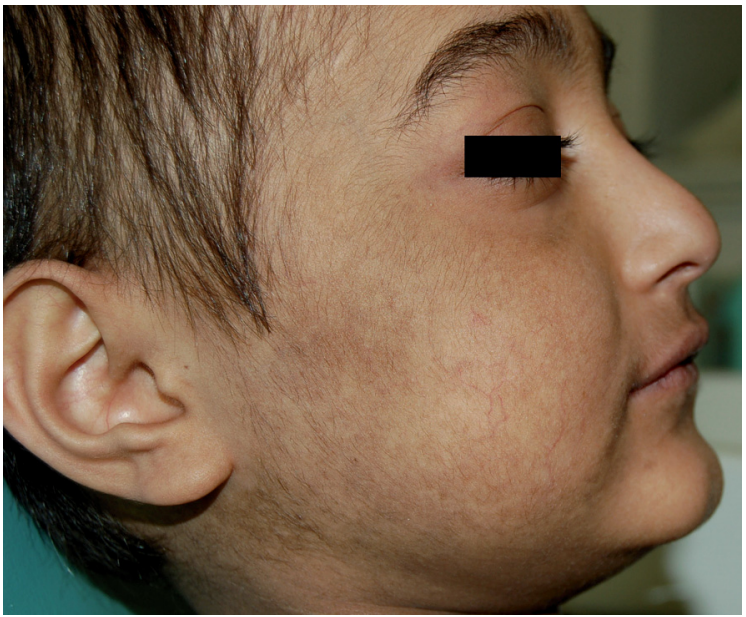

Figure 3 : Aspect exobuccal caractéristique de la thalassémie : protrusion du menton, mélanodermie et alopécie.

Characteristic thalassemic facial appearance: protrusion of chin, melanodermia and alopecia.

Le patient est vu en consultation dans le département d'Odontologie Pédiatrique de Strasbourg en janvier 2006 pour des lésions buccales persistantes. L'anamnèse révèle des antécédents d'avulsions multiples et de soins sur les dents temporaires effectués dans le cadre de la mise en état de la cavité buccale avant la greffe.

L'examen exo-buccal montre un visage de forme carrée avec une proéminence fronto-pariétale, une protrusion des zygomas et du menton, et un élar-

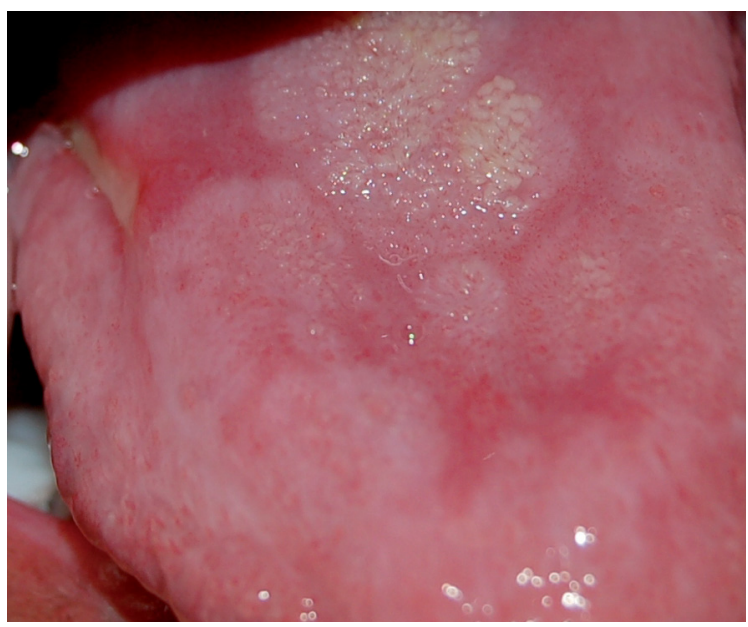

Figure 5 : Lésions linguales lichénoïdes chroniques avec ulcération symptomatique.

Chronic lingual lichenoid lesions with symptomatic ulceration.

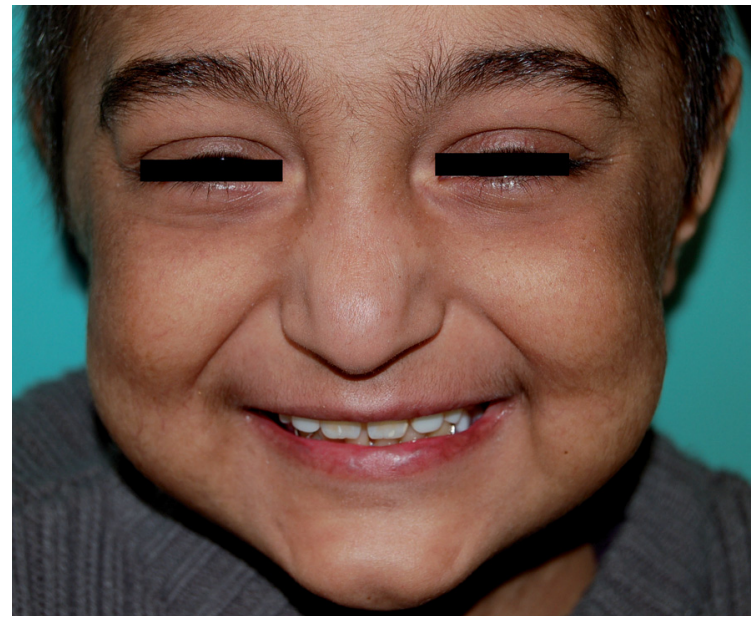

Figure 4 : Aspect exobuccal caractéristique de la thalassémie : visage carré, protrusion zygomatique, proéminence frontale et élargissement du dos du nez. Characteristic thalassemic facial appearance: square face, protrusion of zygoma, frontal prominence and enlargement of the bridge of the nose.

gissement du dos du nez. Le profil de patient est concave avec un sillon labio-mentonnier marqué ; on note une mélanodermie et une alopécie (Fig. 3 et 4). Lors de l'examen endo-buccal, on observe des lésions lichénoïdes chroniques situées sur la langue (Fig. 5) et sur les joues, entourées d'une muqueuse vernissée, d'aspect atrophique. Ces lésions sont le siège d'ulcérations symptomatiques entourées d'une zone érythémateuse (Fig. 5 et 6). Dans la région des commissures labiales, on

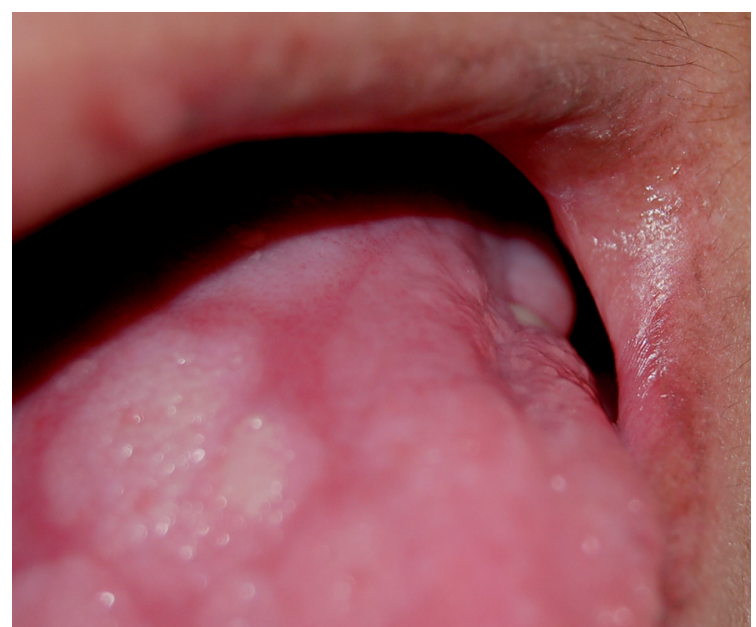

Figure 6 : Ulcération linguale symptomatique, fibrose commissurale.

Symptomatic lingual ulceration, commissural fibrosis. médecine

buccale chirurgie buccale

VOL. $12, N^{\circ} 3$ 2006

page 165 


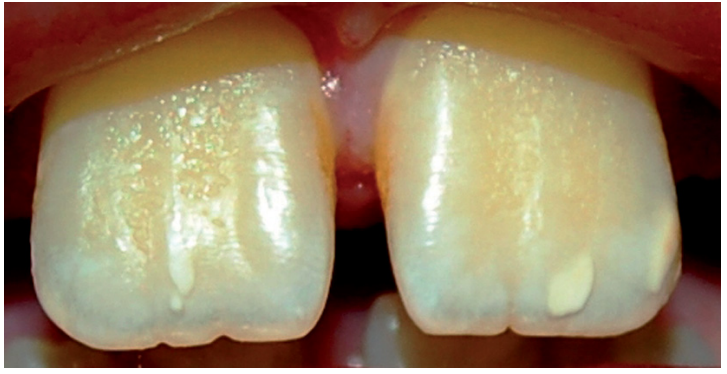

Figure 7 : Dyschromies modérées, diastèmes interincisifs.

Moderate tooth discolorations, inter-incisal diastema.

constate la présence d'une rétraction cicatricielle limitant l'ouverture buccale, associées à des épisodes fréquents de perlèche érosive (Fig. 6). Des dyschromies modérées sont présentes sur l'ensemble des dents (Fig. 7). Une hypertrophie des procès alvéolaires maxillaires est observée (Fig. 8).

médecine

buccale

chirurgie

buccale

VOL. $12, \mathrm{~N}^{\circ} 3$ 2006

page 166 L'examen occlusal montre une relation molaire de classe II, une supraclusion incisive avec morsure palatine, une vestibuloversion incisive, une déviation des milieux et des diastèmes inter-incisifs (Fig. 7).

La radiographie panoramique révèle une radio-opacité sinusienne bilatérale, suggérant une oblitéra-

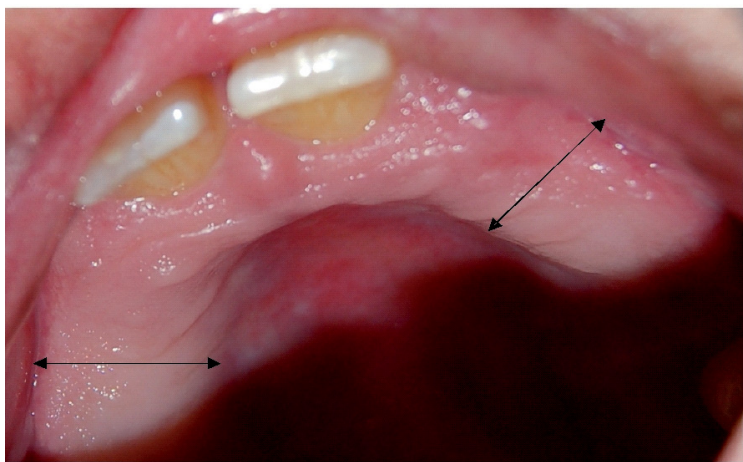

Figure 8 : Elargissement des procès alvéolaires maxillaires.

Enlargement of the maxillary alveolar processes.

tion des sinus maxillaires, et une déviation de la cloison nasale. Les germes de 15 et 25 ont une orientation atypique distale et il existe d'importants espaces entre 36/46 et les germes de 37/47 et entre 36 et les germes de 34 et 35 (Fig. 9). L'examen téléradiographique de profil révèle une absence de pneumatisation du sinus sphénoïde et une morphologie atypique de la selle turcique qui apparaît élargie en raison de l'absence des processus clinoïdes antérieurs. La symphyse mentonnière paraît hypertrophiée (Fig. 10). L'examen céphalométrique par l'analyse de Ricketts confirme la bi-proalvéolie.

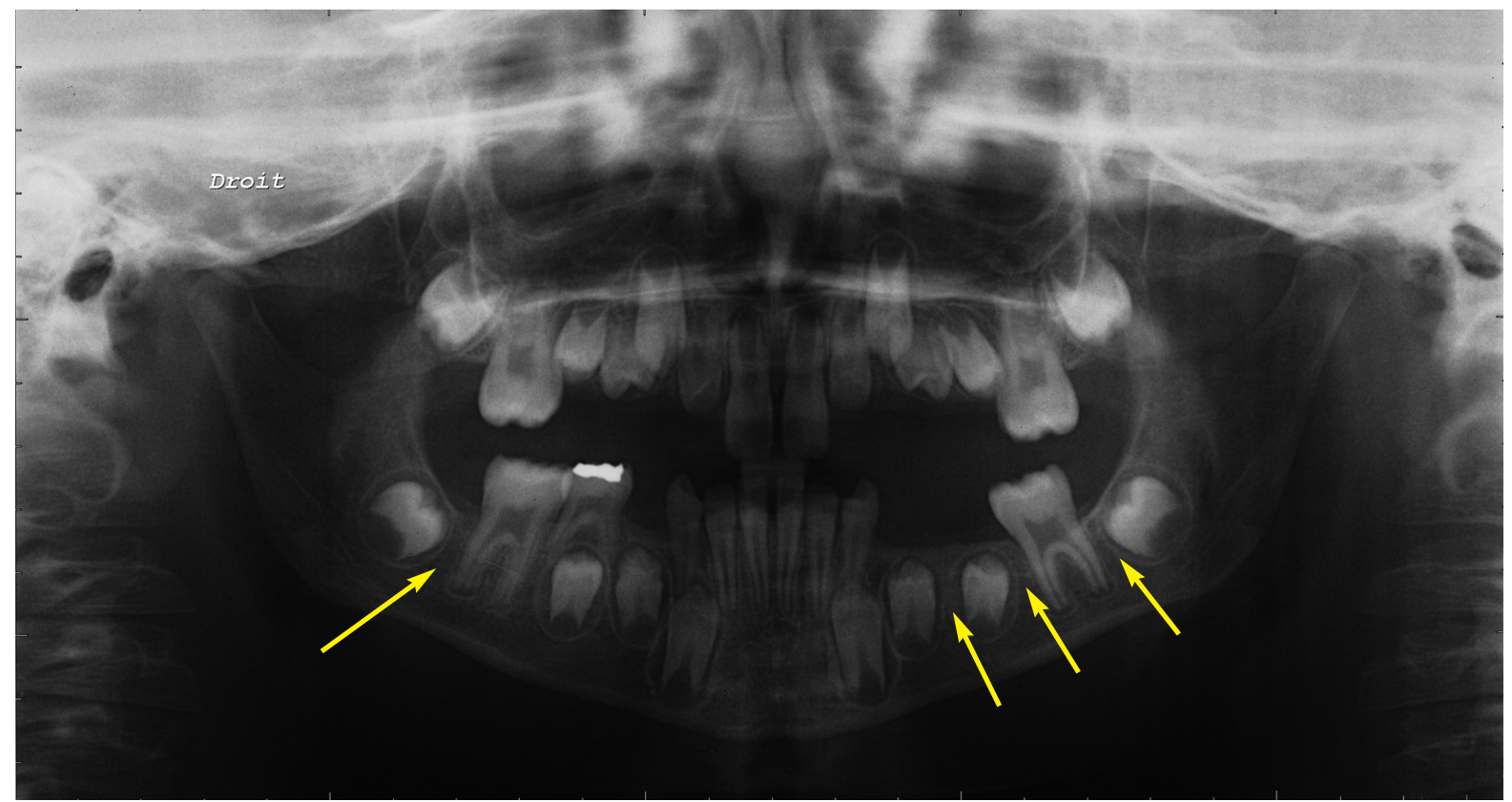

Figure 9 : Orthopantomogramme: oblitération sinusienne, espaces entre les germes. Orthopantomography: sinus obliteration, spaces between germs. 


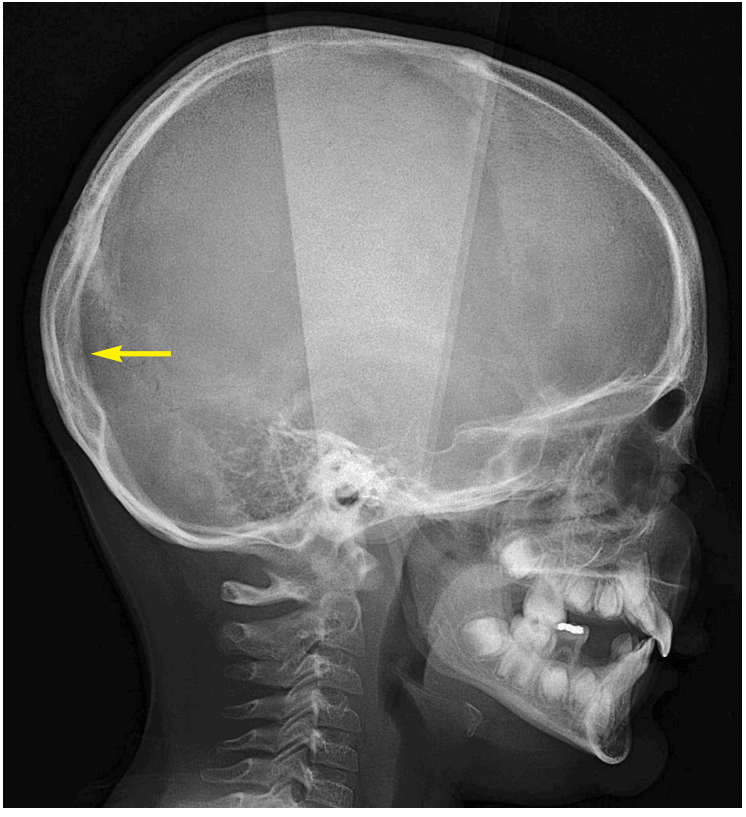

Figure 10 : Téléradiographie de profil : absence d'image en «poils de brosse », absence des processus clinoïdes antérieurs.

Lateral teleradiography: no « hair-on-end » pattern, no anterior clinoid process.

Le patient présente une typologie brachyfaciale avec diminution de la hauteur faciale inférieure ainsi qu'une tendance à la classe II squelettique avec rétrognathie mandibulaire (Fig. 11a et 11b).

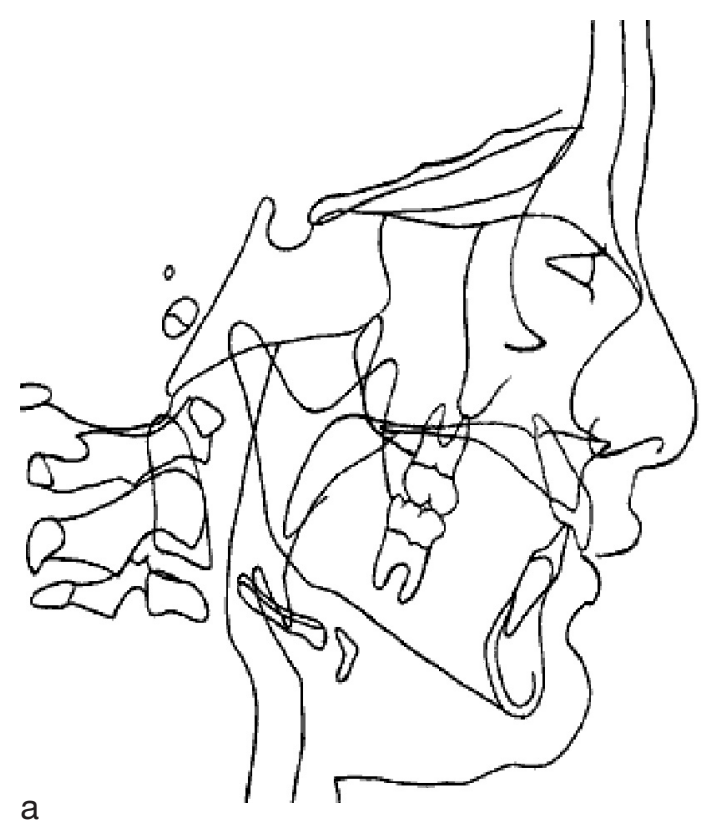

\section{COMMENTAIRE}

Dans la thalassémie majeure, les manifestations osseuses ont un double aspect: il y a d'une part hyperplasie de la moelle hématopoïétique et d'autre part une ostéoporose ${ }^{99}$. La synthèse insuffisante d'hémoglobine se traduit par une importante diminution de la production d'hématies matures. L'hypoxie tissulaire, secondaire à l'anémie, stimule la synthèse d'érythropoïétine. Ce rétrocontrôle positif mène à une expansion médullaire massive intéressant surtout les os de la face et de la voûte crânienne, et certains os longs ${ }^{[10]}$. Cette hyperplasie des espaces médullaires induit le développement de malformations crâniofaciales caractéristiques, retrouvées chez ce patient. II présente un faciès dit thalassémique ou " chipmunck facies » [11] ; le visage est de forme carrée avec une proéminence fronto-pariétale, une protrusion des zygomas et du menton, et un élargissement du dos du nez (Fig. 3 et 4). Ceci explique également l'élargissement des procès alvéolaires maxillaires (Fig. 8). La denture est secondairement déplacée par l'expansion médullaire [10] d'où l'existence de diastèmes inter-incisifs (Fig. 7) et d'espaces entre les germes (Fig. 9). L'ostéoporose, qui est à la fois une conséquence de la croissance des espaces inter-trabéculaires et de la surcharge ferrique, entraîne un amincissement des corticales ${ }^{[9-10]}$. Le traitement précoce par transfusions a limité le développement de ces

\begin{tabular}{|l|cc|c|}
\hline \multicolumn{4}{|c|}{ ANALYSE DE RICKETTS } \\
\hline Axe facial & $90^{\circ}$ & $\pm 3^{\circ}$ & $96^{\circ}$ \\
\hline Plan facial & $87^{\circ}$ & $\pm 3^{\circ}$ & $86^{\circ}$ \\
\hline Angle du plan mandibulaire & $26^{\circ}$ & $\pm 4^{\circ}$ & $23^{\circ}$ \\
\hline Hauteur faciale inférieure & $44^{\circ}$ & $\pm 2,5^{\circ}$ & $39^{\circ}$ \\
\hline Hauteur faciale totale & $60^{\circ}$ & $\pm 3^{\circ}$ & $52^{\circ}$ \\
\hline Arc mandibulaire & $26^{\circ}$ & $\pm 4^{\circ}$ & $27^{\circ}$ \\
\hline Convexité en mm & +2 & \pm 2 & 2 \\
\hline iPt A-Pog en mm & +1 & \pm 2 & 2 \\
\hline iPt A-Pog en degrés & $22^{\circ}$ & $\pm 4^{\circ}$ & $21^{\circ}$ \\
\hline 6 / PTV en mm & 12 & \pm 2 & 13 \\
\hline Lèvre inférieure en mm & +2 & \pm 2 & 6 \\
\hline
\end{tabular}

médecine

buccale

chirurgie

buccale

VOL. $12, \mathrm{~N}^{\circ} 3$ 2006

page 167

Figure 11 a et b : Analyse céphalométrique de Ricketts: classe II, typologie brachyfaciale. Ricketts cephalometric analysis: class II, brachyfacial typology. 
médecine

buccale

chirurgie

buccale

VOL. $12, \mathrm{~N}^{\circ} 3$ 2006

page 168 malformations chez le patient : on ne retrouve pas l'atrophie des corticales, ni l'image typique en «poils de brosse » dans les régions pariétales; bien qu'inconstante, la présence d'une trabéculation perpendiculaire à la voûte crânienne réalise l'aspect du «crâne en brosse», caractéristique de la thalassémie majeure ${ }^{[0,12]}$. La selle turcique présente une forme atypique, évasée, avec absence des processus clinoïdes antérieurs (Fig. 10) ; ces modifications sont secondaires au retentissement de la maladie sur le développement et l'activité sur l'hypophyse. Ceci pourrait aussi expliquer le retard de croissance ${ }^{[9,12] .}$ L'hyperplasie médullaire étant plus importante au maxillaire qu'à la mandibule, les sujets thalassémiques présentent fréquemment des relations squelettiques de classe II avec proalvéolie maxillaire [13-15], ce que confirme l'analyse céphalométrique (Fig. 11a et 11b). La diminution de hauteur faciale totale et de la hauteur faciale inférieure peut s'expliquer par l'absence de pneumatisation des sinus maxillaires mais aussi par l'existence d'édentements multiples non compensés. Un traitement d'orthopédie dento-faciale doit être envisagé après l'éruption complète des dents permanentes et après la fin du traitement immunosuppresseur ${ }^{[16]}$. La classe II peut être corrigée grâce à l'utilisation du « leeway » mandibulaire. En effet, celui-ci est augmenté dans ce cas du fait de l'hyperplasie médullaire qui entraine la formation d'espaces importants entre les germes. Une expansion maxillaire et un mouvement orthodontique de distalisation des premières molaires maxillaires permettront l'éruption de 15 et 25 dont l'orientation est atypique. Un traitement orthopédique fonctionnel à l'aide d'un activateur de croissance mandibulaire viendra éventuellement compléter cette approche. Le traitement médical standard de la thalassémie majeure, qui consiste à réaliser régulièrement des transfusions associées à un chélateur du fer, a permis d'améliorer de manière significative le pronostic à long terme [17]. La greffe de moelle osseuse ou de cellules souches hématopoïétiques issues du cordon ombilical ou du sang périphérique reste le seul traitement curatif de la $B$-thalassémie majeure, surtout pour les patients ayant une faible compliance avec le traitement chélateur ${ }^{[17]}$. Les transfusions multiples entraînent en effet une élévation du fer sérique qui se dépose dans de nombreux organes et tissus ${ }^{\text {(9) }}$ comme en témoignent les dyschromies dentaires (Fig. 7) et la mélanodermie (Fig. 3). L'hémochromatose mettant rapidement en jeu le pronostic vital, ce patient a bénéficié « en urgence » d'une greffe de cellules souches hématopoïétiques prélevées dans le sang périphérique. II a développé rapidement une GVHD après la greffe de cellules provenant d'un donneur non apparenté, ce qui représentait un facteur de risque supplémentaire pour développer une GVHD [18-19].

La GVHD est une complication majeure de la greffe de moelle osseuse allogénique touchant entre 20 et $70 \%$ des patients. Elle se présente sous deux formes (aiguë et chronique) que l'on distingue en fonction de la date de survenue (avant ou après le centième jour post-greffe) et par le tropisme et l'origine des lésions. La GVHD aiguë est une forme transitoire touchant principalement les épithéliums. Cette " allo-réaction ", qui associe classiquement dermatite, hépatite et entérite, est médiée initialement par les lymphocytes $T$ du greffon qui reconnaissent les antigènes du receveur immunosupprimé comme étant du non-soi. La GVHD chronique se traduit par un syndrome évoluant sur un mode récidivant et ubiquitaire, rencontré 3 mois (100 jours) après la transplantation ${ }^{[18,20]}$. Elle possède de nombreuses caractéristiques communes avec certaines maladies auto-immunes, en particulier avec la sclérodermie. En effet, un infiltrat lymphocytaire est retrouvé dans le foie, la peau, la muqueuse buccale et les glandes salivaires ${ }^{[20]}$. Les manifestations buccales les plus fréquentes, présentes chez ce patient, comportent des lésions lichénoïdes, une atrophie muqueuse, des ulcérations (Fig. 5-6), une dysgueusie et une hyposialie [21-22]. Devant l'atteinte de nombreux organes, un traitement immunosuppresseur systémique est nécessaire mais il ne peut pas toujours contrôler les formes extensives [21]. Ce patient a été traité au long cours par une association composée de tacrolimus, de sirolimus et de corticoïdes. II présente une alopécie et une mélanodermie (Fig. 3) qui font certes partie du tableau clinique de la $\beta$ thalassémie mais aussi des effets secondaires de la corticothérapie (syndrome de Cushing). 
Comme il a été montré que les patients atteints de thalassémie majeure présentent un nombre plus important de lésions carieuses [8], les mesures de prévention dentaires classiques (motivation pour l'hygiène bucco-dentaire, applications topiques de fluorures, scellement des sillons des premières molaires) ont été réalisées ; de même qu'une prise en charge spécifique pour la GVHD chronique : applications de xylocaïne visqueuse à $2 \%$ sur les ulcérations jusqu'à 6 fois par jour pendant $30 \mathrm{~s}$, suivies d'un rinçage soigneux pour diminuer les douleurs ${ }^{[23-24]}$ et applications de produits à base de lanoline quotidiennement sur les lèvres ${ }^{[23] .}$ Un traitement antifongique est instauré en cas d'épisode de perlèche. Des applications topiques au niveau des commissures labiales et des bains de bouche à base d'Amphotéricine $B$ (Fungizone $\AA$ ) sont effectués 3 fois par jour jusqu'à résolution complète des lésions candidosiques. Malgré un traitement immunosuppresseur systémique au long cours, il n'y pas eu de réelle amélioration des lésions buccales. L'utilisation de produits immunosuppresseurs topiques a permis d'améliorer l'efficacité sans augmenter la dose et les effets secondaires du traitement systémique [21]. Des bains de bouche à base de dexaméthasone à $0,1 \%$ ont été réalisés 4 à 6 fois par jour [21,23-25], complétés par des applications biquotidiennes de tacrolimus à $0,1 \%$ (Protopic $®)$ [21, 24,26]. Des séances de photothérapie locale (PUVA thérapie), dont le principe repose sur l'exposition de la muqueuse buccale aux ultraviolets $A$ après administration d'un psoralène par voie orale, ont complété cette prise en charge [21,24-25]. Ce traitement, qui provoque l'apoptose des lymphocytes T alloréactifs, comporte peu d'effets secondaires mais entraîne une augmentation à long terme du risque de développement d'un carcinome épidermoïde [21].

Ce traitement requiert un suivi clinique et radiographique régulier et prolongé ${ }^{[16]}$. Une évaluation de la sécrétion salivaire doit être effectuée régulièrement car la GvHD chronique induit une diminution de la sécrétion salivaire à l'origine d'une augmentation du risque carieux [21, 27-28] ; il doit être prévenu par l'application topique de fluorures [24]. Les patients souffrant de GVHD chronique ont une susceptibilité accrue aux infections [18, 27], en particulier d'origine fongique, du fait de l'hyposialie [21] et du traitement immunosuppresseur local. Malgré l'absence de consensus, une antibioprophylaxie est recommandée en cas d'acte invasif [24].

Une surveillance rapprochée ainsi que la réalisation de biopsies sur les lésions lichénoïdes suspectes sont absolument nécessaires du fait du risque accru de transformation maligne en carcinome épidermoïde [20, 24, 29-31].

La multiplicité des manifestations cliniques et des thérapeutiques mises en place illustre la compléxité de l'association entre la ß-thalassémie et les complications de ses traitements que sont I'hémochromatose et la GVHD ; toutes ayant une composante oro-faciale. Le chirurgien-dentiste se doit donc de s'intégrer dans l'équipe multi-disciplinaire prenant en charge les troubles hématologiques.

\section{RÉFÉRENCES}

1 - RUND D, RACHMILEWITZ E. ß-thalassemia. N Engl J Med $2005 ; 353: 1135-46$.

2 - Sodani P, Gaziev D, Polchi P, Erer B, Giardini C, Angelucci E, Baronciani D, Andreani M, Manna M, Nesci S, Lucarelli B, Clift RA, Lucarelli G. New approach for bone marrow transplantation in patients with class 3 thalassemia aged younger than 17 years. Blood 2004 ; 104 : 1201-3.

3 - La Nasa G, Argiolu F, Giardini C, Pession A, Fagioli F, Caocci G, Vacca A, De Stefano P, Piras E, Ledda A, Piroddi A, LitTera R, Nesci S, LOCATELLI F. Unrelated bone marrow transplantation for beta-thalassemia patients: the experience of the italian bone marrow transplant group. Ann NY Acad Sci 2005 ; 1054 : 186-95.

4 - Palencia SI, Rodriguez-Peralto Jl, Castaño E, VANACLOCHA F, IGLESIAS L. Lichenoid nail changes as sole external manifestation of graft versus host disease. Int J Dermatol 2002 ; 41 : 44-5.

5 - EnK CD, Elad S, VeXleR A, Kapelushnik J, Gorodetsky $\mathrm{R}$, KIRSCHBAUM M. Chronic graft versus host disease treated with UVB phototherapy. Bone Marrow Transplant $1998 ; 22$ : 1179-83. médecine

buccale

chirurgie

buccale

VOL. $12, \mathrm{~N}^{\circ} 3$

2006

page 169 
6 - Chib FJ, Ratnavel R, Samson D, Apperley J, BaL J, Taylor P, RUSSELL-JONES R. Extracorporeal photopheresis (ECP) in the treatment of chronic graft versus host disease (GvHD). Bone Marrow Transplant 1999 ; 23 : 881-7.

7 - Couriel D, Hosing C, Saliba R, Shpall EJ, Andelini P, POPAT U, DONATO M, Champlin R. Extracorporeal photopheresis for acute and chronic graft versus host disease: does it work? Biol Blood Marrow Transplant $2006 ; 12: 37-40$.

8 - Al-Wahadni AM, TaAnI DQ, Al-OMari MO. Dental diseases in subjects with B-thalassemia major. Community Dent Oral Epidemiol 2002 ; 30 : 418-22.

9 - SOMMERMATER JI, BACON W. Manifestations dento-maxillaires et maxillo-faciales de la thalassémie majeure : à propos d'un cas. Act Odont Stomatol 1981 ; 135 : 489-99.

10 - DREW SJ, SACHS SA. Management of the thalassemiainduced skeletal facial deformity: case reports and review of the literature. J Oral Maxillofac Surg 1997 ; $55: 1331-39$.

11 - DugGal MS, Bedi R, Kinsey SE, Williams SA. The dental management of children with sickle cell disease and ß-thalassemia: a review. Int J Paediatr Dent 1996; 6 : 227-34.

médecine buccale chirurgie buccale

VOL. $12, \mathrm{~N}^{\circ} 3$ 2006

page 170
22 - Treister NS, Woo SB, O'Holleran EW, Lehmann LE, PARSONS SK, GUINAN EC. Oral chronic graft versus host disease in pediatric patients after hematopoietic stem cell transplantation. Biol Blood Marrow Transplant 2005 ; $11: 721-31$.

23 - CAmeron AC, Widmer RP. Appendices: Oral management of the pediatric bone marrow transplant patient (pp 397-401). In: Handbook of Pediatric Dentistry, 2nd ed. Mosby, Sydney, 2003.

24 - Couriel D, Carpenter Pa, Cutler C, Bolanos-Meade J, Treister NS, Gea-Banacloche J, Shaughnessy P, Hymes S, Kim S, Wayne AS, Chien JW, Neumann J, Mitchell S, Syrjala K, Moravec CK, Abramovitz L, Liebermann J, Berger A, Gerber L, Schubert M, Filipovich AH, WeIsdorf D, Schubert MM, Shulman H, Schultz K, Mittelman B, Pavletic S, Vogelsang GB, MARTIN PJ, LeE SJ, Flowers ME. Ancillary therapy and supportive care of chronic graft versus host disease: National Institutes of health consensus development project on criteria for clinicals trials in chronic graft versus host disease: V. Ancillary therapy and supportive care working group report. Biol Blood Marrow Transplant 2006 ; $12: 375-96$.

25 - WolfF D, ANders V, Corio R, Morison WL, FARmer E, VOGELSANG GB. Oral PUVA and topical steroids for treatment of oral manifestations of chronic graft versus host disease. Photodermatol Photoimmunol Photomed $2004 ; 20$ : 184-90.

26 - Eckardt A, Starke O, Stadler M, Reuter C, HeRTENSTEIN B. Severe oral chronic graft versus host disease following allogenic bone marrow transplantation: highly effective treatment with topical tacrolimus. Oral Oncol $2004 ; 40: 811-4$.

27 - Majorana A, Schubert MM, Porta F, Ugazio AG, SAPELLI PL. Oral complications of pediatric hematopoietic cell transplantation: diagnosis and management. Support Care Cancer $2000 ; 8$ : 353-65.

28 - NAGLER RM, NAGLER A. Salivary gland involvement in graft versus host disease: the underlying mechanism and implicated treatment. IMAJ 2004 ; 6 :167-72.

29 - Bahtia AD, Louie AD, Bahtia R, O'Donnel MR, Fung H, Kashyap A, Krishnan A, Molina A, Nademanee A, Niland JC, Parker PA, Snyder DS, Spielberger R, StEIN A, Forman SJ. Solid cancers after bone marrow transplantation. J Clin Oncol 2001; 19 : 464-71.

30 - Abdelsayed RA, Sumner T, Allen CM, Treadway A, NEss GM, PENZA SL. Oral precancerous and malignant lesions associated with graft versus host disease: Report of 2 cases. Oral Surg Oral Med Oral Pathol Oral Radiol Endod 2002 ; 93 : 75-80.

31 - SZETO CH, SHEK TWH, LIE AKW, Au WY, YUEN APW, KWONG YL. Squamous cell carcinoma of the tongue complicating chronic oral mucosal graft versus host disease after hematopoietic stem cell transplantation. Am J Hematol 2004 ; 77 : 200-2. 